\title{
Application of Biological Detection Technology in Food Inspection
}

\author{
Zhang Lihua \\ ChiFeng Indusrty Vocational Technology College, 024005
}

\begin{abstract}
Food safety issues are related to people's livelihood issues and are deeply valued by the society. They affect the hearts and minds of hundreds of millions of people. In recent years, food safety issues have been frequent, and government departments, food processing enterprises and private consumer organizations should work together to strengthen and pay attention to food inspection, in order to ensure food supply quality, maintain food safety, and develop healthy lifestyles. Therefore, how to efficiently and accurately detect the harmful substances contained in food has become a key topic in food quality monitoring. As a new technology field in the 21 st century, bioassay technology has unique advantages in food safety monitoring. Explain the application of bio-inspection technology in food inspection, in order to providing reference for relevant personnel and promote the rapid development of food testing industry.
\end{abstract}

\section{Introduction}

With the rapid development of the food industry, the importance of food safety testing technology is increasing day by day. The traditional food safety testing methods are inefficient and inaccurate, and it is difficult to adapt to the needs of the development of the times. The development of science and technology has promoted the application of biotechnology. It has the advantages of simplicity, high sensitivity, high specificity, etc. The development of science and technology has a good prospect in the food safety testing industry. In the past, China's food regulatory authorities and consumers have not paid enough attention to food safety, food safety supervision is not strict, food safety testing system is not perfect, food safety testing technology is not scientific and advanced, resulting in frequent food safety accidents, foods often appear Toxic and harmful substances pose a threat to human health. Therefore improve the importance of food safety and quality testing, and continuously develop food safety testing technology is of great significance.

\section{Overview of the Application of Biotechnology in Food Testing}

The application of biotechnology in the food field has a long history. Bread, soy sauce, yoghurt, kimchi... The production of these foods is inseparable from the application of biotechnology. Nowadays, the application of biotechnology in the food field has gradually extended to food. The various fields of science have been fully developed in the continuous improvement. Based on basic disciplines such as cell biology, modern biotechnology has developed into a variety of biotechnology including genetic engineering, cell engineering, enzyme engineering, and fermentation engineering. In the field of food science, bio-organisms are used to synthesize, transform, and degrade biological organisms to obtain product production. Among them, genetic engineering is to change the characteristics of organisms by recombining them to obtain special foods; cell engineering is based on the technology of animal and plant cells and cell fusion to produce food; enzyme engineering is to use active substances in living organisms - - Enzymes, which convert ingredients in foods and change food traits; fermentation engineering is a traditional food production process that uses fermentation technology to improve food traits and obtain foods of different tastes [1].

\section{Overview of the Application of Molecular Biology in Food Testing}

\subsection{Gene Chip Technology}

Genechip, also known as DNA chip and biochip, is a modern biotechnology method proposed in the 1980s. The principle of the gene chip is hybridization sequencing method - immobilizing a set of target nucleotide probes of known sequence on the surface of a substrate, fluorescently labeling the nucleic acid sequence in the solution, and then continuously complementing the known sequence on the substrate. Pairing, when the fluorescent signal is strongest, obtains a set of probe sequences which are completely complementary to the known sequence by identifying the position of the probe, thereby determining the gene sequence of the substance in 
the solution. In this way, we can understand the genetic sequence of the ingredients in the food.

When genetically modifying foods, it is necessary to understand the role of genes in the life processes, especially the morphological characteristics of organisms. Traditional biotechnology methods are difficult to understand the role of such a large number of genes in life processes. Gene chip technology has largely solved this problem. Gene chip technology immobilizes a large number of probe molecules $(>400$ cells $/ \mathrm{cm} 2)$ on a substrate while pairing with fluorescently labeled sample genes, typically wafers or slides. Such a substrate is very similar to a computer's electronic chip. Therefore, such a technology is called gene chip technology. Genetic chip detection with gene chip technology is fast, efficient, and highly automated.

The widespread use of gene chip technology is inseparable from the development of probe solid phase in situ synthesis and photolithography, as well as the application of laser confocal microscopy. The probe solid phase in situ synthesis technology enables the synthesis of high-density probe molecular chips, and the laser confocal microscopy technology enables real-time, sensitive and accurate detection of hybridization signals. In the process of food safety testing, the application of gene chip technology can detect all potential pathogens at one time, comprehensive, efficient and accurate [2].

\subsection{Gene Probe Technology}

Gene probe technology, also known as molecular hybridization, is a technique for achieving specific DNA probes based on the specificity of base-pair pairing. The process of pairing DNA single strands into double strands is specific, and only two complementary strands of DNA can be combined to form a double strand, ie, capable of hybridization. At the same time, this hybridization can occur not only between DNA but also between DNA and RNA. For gene probe detection, two conditions must be met-specific DNA probes and genomic DNA. After the two are processed into single strands, hybridization can begin. A gene probe is a nucleic acid sequence complementary to a gene of interest. It can be a complete gene or a gene fragment; it can be DNA or RNA transcribed from DNA. According to the source of the gene probe, the gene probes can be divided into three categories: 1 the DNA of the genome itself, called a genomic probe; 2 , first transcribe the mRNA from the genome, and then reverse-transcribe the mRNA to obtain a cDNA probe, such a cDNA The probe does not contain an intron sequence; 3 an oligonucleotide probe complementary to the gene sequence with a small number of bases synthesized in vitro. By labeling the probe, a signal can be acquired at the position where the probe hybridizes to the genomic DNA, usually using the radioisotope $32 \mathrm{P}$ to label the alpha phosphate group on the nucleotide [3].

\section{Overview of the Application of Immunology in Food Testing}

\subsection{Immunofluorescence Technique}

Immunofluorescence technology is a combination of immunological techniques and fluorescent labeling techniques to study the distribution of specific protein antigens in cells. The immunofluorescence technique firstly fluorescently labels a known antigen or antibody by fluorescein, and then uses a fluorescence microscope to search for the antigen or antibody to determine an antigen or antibody in the tissue or the cell to achieve quantitative or qualitative analysis of the antigen or antibody properties. Among them, a method of tracing with a fluorescent antibody is called a fluorescent antibody method, and a method of tracing using a fluorescent antigen is called a fluorescent antigen method, and the two methods are collectively referred to as an immunofluorescence technique. The method of detecting antigens or antibodies in tissues or cells using immunofluorescence techniques is called immunofluorescent cell (or tissue) chemical technology. Common immunofluorescence cytochemical methods include direct method, sandwich method, indirect method and complement method. The specific operation procedure of the immunofluorescence technique is to firstly pair the sample sample to be detected and the fluorescently labeled antibody or antigen with the antibody antigen on the surface of the substrate, and then wash and separate the antibody antigen complex on the substrate, and finally wait for the substrate. After the bound enzyme is proportional to the amount of the test component in the specimen, the substrate of the enzyme reaction is added, and the substrate is catalyzed by the enzyme into a colored substance, because the amount of the colored product is positively correlated with the amount of the substance to be tested in the specimen, and therefore, The content of the substance to be tested in the sample is determined according to the depth of dyeing.

\subsection{Enzyme-linked Immunosorbent Assay}

The enzyme-linked immunosorbent assay is an immunoassay technique that combines the specificity of an antigen-antibody reaction with the efficiency and specificity of an enzyme-catalyzed substrate reaction using an enzyme-labeled antibody (antigen) as a main reagent. This is essentially a combination of immunofluorescence technology and radioimmunoassay technology. First, the antibody or antigen is adsorbed on a substrate, and subjected to immunoenzymatic staining. After the substrate is developed, qualitative or quantitative analysis is performed. Enzymes commonly used in enzyme-linked immunosorbent assays include horseradish peroxidase (HRP) and alkaline phosphatase (AP); common substrates include o-phenylenediamine (OPD), tetramethylbenzidine (TMB), and sodium nitrate. Benzoyl phosphate (p-NPP). Enzyme-linked immunosorbent assay is essentially a combination of immunofluorescence technology and radioimmunoassay. There are many branches, including indirect methods, double antibody sandwich method, capture method, competition method, and enzyme-linked immunosorbent assay for antigen and antibody testing. Simultaneous 
qualitative and quantitative analysis, high sensitivity, wide application range, strong stability, simple and fast, low cost, fast speed, can detect thousands of samples at the same time, highly respected [4].

\section{Overview of the Application of Metabolism in Food Testing}

\subsection{Impedance Method}

Impedance method is the process of using microorganisms to metabolize non-electroactive substances in the medium into electrically active substances, so that the conductivity is the medium is improved and the impedance of the material in the medium is lowered. In order to identify bacteria in the medium and to understand the reproduction of the bacteria, there are many impedance in the medium. The impedance method has high sensitivity, specificity, rapid response and high reproducibility. It is often used in food quality and pathogen detection, clinical sample bacterial detection, microbial process control in industrial production, and environmental sanitation bacteriology.

\subsection{Radiometry}

Radioactivity measurement is a new diagnostic technique based on physics and chemistry. It is used to put radioactive $\mathrm{C}$ element into the growth and reproduction medium of bacteria. During the breeding process, the bacteria can metabolize radioactive $\mathrm{C}$ element to $\mathrm{CO}$ containing radioactive $\mathrm{C}$, and the radioactivity can be measured by the instrument. The presence and content of $\mathrm{CO}$ change to determine the presence or absence of bacteria in the medium. Radiometric method is highly accurate, fast and highly automated, suitable for bacterial detection and sterility identification of large samples.

\section{Polymerase Chain Reaction (PCR)}

Polymerase chain reaction (PCR) can be used to amplify specific DNA fragments, and PCR technology can be considered as in vitro DNA replication. A small amount of DNA can be rapidly and massively copied and amplified using PCR technology. Using PCR technology, the DNA extracted from the murder scene or trace DNA in ancient fossils can be rapidly amplified, aligned and identified. PCR technology was invented by Mullis in the United States in 1985. By 2013, PCR technology has undergone three generations of development. In 1976, the stable Taq DNA polymerase discovered by Chinese scientist Qian Jiayun was made for the advancement of PCR technology. A fundamental contribution. The DNA is detached from the organism and can be dissociated into a single strand at a high temperature of $95^{\circ} \mathrm{C}$. At a low temperature of about $60^{\circ} \mathrm{C}$, the primer can be bound to the DNA single strand by base pairing. Thereafter, the temperature is adjusted to the DNA polymerase. After a suitable reaction temperature of about $72^{\circ} \mathrm{C}$, the DNA polymerase will form a complementary strand along the direction of phosphoric acid to five carbon sugars. In fact, the PCR instrument is a temperature-controlled device that regulates temperature at various stages of DNA dissociation, denaturation, and replication. PCR technology is also called cell-free cloning system. With this detection method, the detection speed is fast, the sensitivity is high, the specificity is high, and the detection cost is low, but the problem of PCR product contamination is difficult to control.

\section{7."Dry Film" Method}

The "dry film" method uses a non-toxic polymer material as a medium carrier to quickly detect microorganisms in test paper and film. The "dry film" method for quantitative detection technology is very mature, and its high precision and accuracy can be compared with standard methods for some samples. The "dry film" method for the determination of microorganisms does not require reagents, the operation is simple and rapid, the sample storage is easy, the transportation and carrying are convenient, the cost is low, and it can be carried out anytime and anywhere. The detection process only needs "dry film", does not produce waste waste liquid, and has less harm to the environment. It is suitable for microbial measurement in various environments such as field environment, production site and laboratory. While ensuring a higher quality of inspection, the labor intensity is greatly reduced.

\section{Constant Temperature Amplification}

In recent years, with the rapid development of molecular biology technology, diagnostic methods based on nucleic acid detection have been widely established and widely used in laboratory testing of animal diseases, and constant temperature amplification technology has emerged in this context. Compared to other nucleic acid amplification techniques, constant temperature amplification has the advantages of being fast, efficient, and specific, and requires no special equipment. Therefore, it has been considered by many scholars as a detection method that is likely to be comparable to PCR. At present, the main constant temperature amplification techniques are: rolling circle amolification (RCA), loop-mediated isothermal amplification ( Loop-mediated isothermal amplification (LAMP), strand displacement amplification (SDA), nucleic acid sequence based amplification (NASBA) and helix dependent amplification (HAD) [5].

\section{Biochip Technology}

Biochip refers to the method of in situ synthesis or microspotting of light guides to form a large number of biological macromolecules such as nucleic acid fragments, polypeptide molecules or even tissue sections, cells and other biological samples on the surface of the support in an orderly manner. The dense two-dimensional molecular arrangement is then hybridized with the target molecule in the labeled biological sample to be tested, and the intensity of the hybridization signal is detected and 
analyzed rapidly, in parallel, and efficiently by a specific instrument to determine the number of target molecules in the sample. Since the conventional silicon wafer is used as a solid phase support and the preparation technology of the computer chip is simulated in the preparation process, it is called a biochip technology. Intracellular mRNA or reverse transcription from different individuals (normal humans and patients), different tissues, different cell cycles, different developmental stages, different differentiation stages, different lesions, different stimuli (including different induction, different treatment stages) The post-production cDNA hybridizes with the expression profile gene chip, and can comprehensively analyze and judge the individual specificity, tissue specificity, developmental stage specificity, differentiation stage specificity, lesion specificity and stimulation specificity of these gene expressions. Quickly linking one or several genes to disease greatly accelerates the establishment of these genes and further studies the relationship between genes and genes. Therefore, no matter what kind of research field, gene expression chip can be used to obtain a large number of genes related to the research field, making the research more purposeful and systematic, and also broadening the research field [5].

\section{Conclusion}

The continuous application of biotechnology in food testing in China has been recognized by researchers and has become a widely used method in food testing. With the development of China's science and technology, with the joint efforts of researchers from all sides, the application of biotechnology in food testing will be more mature, laying a technical foundation for China's food safety.

\section{References}

1. Xialapati Azati. Application of bioassay technology in the field of food inspection [J]. Modern food, 2018 (06): 8-9+13.

2. Wei Chao. Application analysis of bio-detection technology in food inspection [J]. SME Management and Technology (late issue), 2017 (06): 176-177.

3. Gu Chengpeng, Yu Hua, Xu Xia, Yuan Hefang. Application of Bioassay Technology in Food Inspection[J].Food Safety Guide,2016(12):38.

4. Ye Li Nur, Hamuza, Xiong Suyu. Discussion on the Application of Bioassay Technology in Food Inspection[J].Biochemical,2016,2(02):54-55.

5. Dong Zhigang. Application of Biotechnology in Food Inspection [J]. Food Safety Guide, 2019 (14): 12-13. 